\title{
On the number of connected sets in bounded degree graphs*
}

\author{
Kustaa Kangas Petteri Kaski Janne H. Korhonen \\ Department of Computer Science \\ Aalto University \\ Finland \\ \{juho-kustaa.kangas, petteri.kaski, janne.h.korhonen\}@aalto.fi \\ Mikko Koivisto \\ Department of Computer Science \\ University of Helsinki \\ Finland \\ mikko.koivisto@helsinki.fi
}

Submitted: Nov 10, 2017; Accepted: Aug 27, 2018; Published: Nov 16, 2018

(c) The authors. Released under the CC BY-NC-ND license (International 4.0).

\begin{abstract}
A set of vertices in a graph is connected if it induces a connected subgraph. Using Shearer's entropy lemma and a computer search, we show that the number of connected sets in a graph with $n$ vertices and maximum vertex degree $d$ is at most $O\left(1.9351^{n}\right)$ for $d=3, O\left(1.9812^{n}\right)$ for $d=4$, and $O\left(1.9940^{n}\right)$ for $d=5$. Dually, we construct infinite families of graphs where the number of connected sets is at least $\Omega\left(1.7651^{n}\right)$ for $d=3, \Omega\left(1.8925^{n}\right)$ for $d=4$, and $\Omega\left(1.9375^{n}\right)$ for $d=5$.

Mathematics Subject Classifications: 05C07, 05C30, 05C35, 05C40, 68R05, $68 \mathrm{R} 10$
\end{abstract}

\section{Introduction}

A connected set in an undirected graph is a subset of vertices that induces a connected subgraph. Besides being fundamental combinatorial objects, connected sets play a key role in various exponential-time graph algorithms. For instance, for an $n$-vertex graph one can solve the traveling salesman problem [7], solve the maximum internal spanning tree

*A preliminary version of this work was presented at the 40th International Workshop on GraphTheoretic Concepts in Computer Science [22]. The work was supported by the European Research Council, under Grant 338077, and by the Academy of Finland, under Grant 276864. 
problem [4], and evaluate the Tutte polynomial [5] in time that is within an $n^{O(1)}$ factor of the number of connected sets of the graph. Within the same time bound an algorithm also finds an optimal Bayesian network having the input graph as its super-structure [26].

Relatively little is known about extremal combinatorics of connected sets in different graph classes. What is immediate, however, is that an $n$-vertex graph can have at most $2^{n}$ connected sets and that this bound is achieved by complete graphs. It is also easy to see that sparsity alone does not imply a much smaller number of connected sets: an $n$-star has an average degree less than 2 , but the number of connected sets is $2^{n-1}+n$. In this light, graphs of bounded degree form a natural graph class to study; we define the degree of a graph as the maximum degree of a vertex. Parameterizing by the size of the connected set, Bollobás [8, pp. 129-130] provides two ways to prove that any graph of degree $d \geqslant 3$ has at most $(\mathrm{e}(d-1))^{k}$ connected sets with $k+1$ vertices, one of which is a given vertex. For large $k$, this bound is, however, loose and of no use for bounding the total number of connected sets of an $n$-vertex graph. The first nontrivial upper bound, namely $\beta_{d}^{n}+n$, where $\beta_{d}=\left(2^{d+1}-1\right)^{1 /(d+1)}$, was given by Björklund et al. [7]. In particular, we have $\beta_{d}=1.9680,1.9874,1.9948$ for $d=3,4,5$, respectively. We are not aware of better bounds, prior to this work.

There is no reason to believe that the Björklund et al. bound is tight. First, its proof applies Shearer's entropy lemma, in essence, by taking the product of the number of possible projections of connected sets to the closed neighborhood of each vertex. Specifically, the proof provides no means to construct a graph that would attain the upper bound. Also, connectivity is an inherently global property that cannot be captured by looking at individual local neighborhoods. Second, while it is easy to construct arbitrarily large graphs that have an exponential number of connected sets, getting near the upper bounds appears to be challenging.

In this paper, we seek improved upper bounds for the number of connected sets by applying Shearer's entropy lemma in an expanded context. Namely, we are interested in projecting the connected sets not only to the immediate closed neighborhood of each vertex but rather to the ball of radius $r \geqslant 2$ (the $r$-neighborhood) around each vertex. By carrying out a computer search over the possible projections of connected sets to $r$-neighborhoods, we obtain improved upper bounds for $d \leqslant 5$.

Theorem 1. Every graph on $n$ vertices with maximum degree $d \leqslant 5$ has at most $b_{d}^{n}+n\left(2^{d^{2}+1}-1\right)$ connected sets, where $b_{3}=1.9351, b_{4}=1.9812$, and $b_{5}=1.9940$.

Dually, we show the following lower bounds for $d \leqslant 5$.

Theorem 2. For each $d \leqslant 5$ there is an infinite family of graphs with maximum degree $d$ where each graph of $n$ vertices has at least $\Omega\left(a_{d}^{n}\right)$ connected sets, where $a_{3}=1.7651$, $a_{4}=1.8925$, and $a_{5}=1.9375$.

These lower bounds improve upon our preliminary results, which were obtained by analyzing families of generalized ladder graphs [22]. The new lower bounds are due to similar constructions where multiple "gadgets" of low degree are chained together, admitting a simple recursive characterization of the number of connected sets. We expect 
each of these bounds to be improved further by expanding the search over the space of possible gadgets. The main obstacle here is the computational cost of analyzing a single gadget, as it scales exponentially in the number of vertices of the gadget. The programs and gadgets used to prove Theorems 1 and 2 are available in the online appendix. ${ }^{1}$

Finally, since we are not aware of any non-trivial lower bounds for graphs of arbitrary degree in the literature, we present one such bound, based on a certain parameterized family of the gadget constructions.

Theorem 3. For each $d \geqslant 6$ there is an infinite family of graphs where each graph of $n$ vertices has at least $\Omega\left(a_{d}^{n}\right)$ connected sets, with $a_{d}=\left(3 \cdot 2^{d-5}\right)^{1 /(d-3)}$.

Related work. The maximum number of subsets of vertices satisfying a given property has been studied for many different types of properties. In the case of maximal independent sets (or dually, maximal cliques), the classical Moon-Moser $[25,23]$ upper bound is known to be tight. Tight upper bounds have also been established in the case of maximal induced matchings and other families of maximal induced $r$-regular subgraphs [20]. In the case of maximal bicliques [15], the known upper bound is tight up to a polynomial factor. For various other important properties the gap between the known lower and upper bounds remains relatively large, such as in the case of minimal dominating sets [12], minimal connected dominating sets [18], minimal feedback vertex sets (in general graphs [11] or in tournaments $[24,17])$, minimal independent feedback vertex sets [1], minimal connected vertex covers [19], maximal induced bipartite subgraphs [9], minimal separators [16], potential maximal cliques $[13,16]$, maximal induced $d$-degenerate subgraphs [27], and irredundant sets [3]. In a typical case the upper bound is obtained by a careful analysis of an appropriate branching algorithm.

Entropy methods, applied in the present work, have previously yielded tight bounds for certain properties in bounded degree graphs. For independent sets in $d$-regular graphs with $n$ vertices the bound $\left(2^{d+1}-1\right)^{n / 2 d}$ was conjectured to be tight by Alon [2]. Kahn [21] showed that the conjecture holds for bipartite graphs. Later, Zhao [29] confirmed that the conjecture holds in general by presenting an unexpectedly simple reduction to the bipartite case; Galvin [14] reviews earlier developments. Björklund et al. [6] used Shearer's entropy lemma to show that the bound $\left(2^{d+1}-1\right)^{n /(d+1)}$ is tight for the number of dominating sets in $n$-vertex graphs of degree at most $d$.

In the case of connected sets, Björklund et al. [7] give further upper bounds for the number of connected sets when the connected sets are also required to be dominating or "transient" (a more strict condition that any prefix of a Hamiltonian cycle must satisfy), or when the graph is assumed to be triangle-free. In particular, the traveling salesman problem can also be solved within an $n^{O(1)}$ factor of the number of transient connected sets, improving upon the bound based on connected sets alone. Motivated by an application to structure learning in Bayesian networks, Perrier, Imoto, and Miyano [26] present an empirical study on the number of connected sets in random bounded degree graphs. Vince [28] gives degree-based conditions under which the number of connected sets grows exponentially, as well as tight bounds in terms of the number of edges.

\footnotetext{
${ }^{1}$ https://github.com/jwkangas/connected-sets.
} 


\section{Upper Bounds on the Number of Connected Sets}

Our upper bounds are derived by extending the Shearer's entropy lemma -based projection approach of Björklund et al. [7] to consider neighborhoods whose radius $r$ is greater than one. Here the essential difficulty and our contribution is to develop computer-assisted analytical tools to study projections of connected sets to neighborhoods of vertices.

We begin by reviewing a basic template suitable for any maximum degree $d$ and any radius $r$ for vertex neighborhoods. We then proceed to characterize in more detail the worst-case graphs induced by the neighborhoods. Making use of the characterization, we give an algorithm that suffices to carry out a complete analysis of the cases $d \leqslant 5$ and $r \leqslant 2$, leading to Theorem 1 . While a computer search would be feasible beyond these parameters, we conclude this section by showing that our method of studying the "boundary-connected" projections appears to be restricted to the case $d \leqslant 5$ and $r \leqslant 2$. That is, beyond these parameters, an analysis based on boundary-connectivity appears not to yield improved upper bounds over those obtained by simply taking $r=1$.

\subsection{The Projection Method}

Our main tool for deriving upper bounds for the number of connected sets is Shearer's entropy lemma, which is most conveniently deployed in our context in the following combinatorial form:

Lemma 4 (Chung et al. [10]). Let $V$ be an n-element set and let $A_{1}, A_{2}, \ldots, A_{k}$ be subsets of $V$ such that every $v \in V$ occurs in at least $\delta$ of these subsets. Let $\mathcal{F}$ be a set of subsets of $V$. For each $1 \leqslant i \leqslant k$, define the projections $\mathcal{F}_{i}:=\left\{F \cap A_{i}: F \in \mathcal{F}\right\}$. Then,

$$
|\mathcal{F}|^{\delta} \leqslant \prod_{i=1}^{k}\left|\mathcal{F}_{i}\right|
$$

Lemma 4 enables us to obtain control over the number of connected sets in a graph by taking the sets $A_{i}$ to be (augmented) neighborhoods of vertices.

In more precise terms, let $G$ be an undirected graph with vertex set $V$ and let $S \subseteq V$ be a subset of vertices. Let $r=0,1, \ldots$ be a radius parameter. Let us write $N_{G}^{r}[S]$ for the set of all vertices $u \in V$ such that there exists a vertex $v \in S$ for which the shortest-path distance between $u$ and $v$ is at most $r$. In particular, when $S=\{v\}$ is a singleton set consisting of the vertex $v \in V$ only, we write $N_{G}^{r}[v]$ for $N_{G}^{r}[S]$ and say that $N_{G}^{r}[v]$ is the (closed) neighborhood of the vertex $v$ of radius $r$. When $r=1$ we may omit the parameter $r$ from the notation. We observe that $N_{G}^{0}[S]=S$ and that $N_{G}^{r}[S]=N_{G}\left[N_{G}^{r-1}[S]\right]$ for $r \geqslant 1$.

The following immediate lemma recalls the Moore bound $\delta_{r}$ for the size of $N_{G}^{r}[v]$.

Lemma 5. Suppose that the graph $G$ has maximum vertex degree $d$. Then for all $r=$ $0,1, \ldots$ and all vertices $v \in V$ it holds that $\left|N_{G}^{r}[v]\right| \leqslant \delta_{r}$, where

$$
\delta_{r}:=1+d \sum_{i=0}^{r-1}(d-1)^{i}=\frac{d(d-1)^{r}-2}{d-2} .
$$


Now let $\mathcal{F}$ be a set of subsets of $V$. For $r=0,1, \ldots$ and $v \in V$, let us write $\mathcal{F}_{v, r}=$ $\left\{F \cap N_{G}^{r}[v]: F \in \mathcal{F}\right\}$ for the projection of $\mathcal{F}$ into the neighborhood of $v$ of radius $r$. We are now ready to prove our main template for upper bounds. In essence, this lemma replaces the application of Jensen's inequality in the Björklund et al. [7] analysis with a uniform bound (the parameter $\rho$ ) that is easier to deploy over larger neighborhoods.

Lemma 6. Let $0 \leqslant \rho \leqslant 1$ be a number such that $\left|\mathcal{F}_{v, r}\right| \leqslant 2^{\left|N_{G}^{r}[v]\right|} \rho$ holds for all $v \in V$. Then, $|\mathcal{F}| \leqslant\left(2 \rho^{1 / \delta_{r}}\right)^{n}$.

Proof. Our intent is to apply Lemma 4. Towards this end, start by setting $A_{v}:=N_{G}^{r}[v]$ for each $v \in V$. Next, for each $u \in V$, if $u$ is contained in $k \leqslant \delta_{r}-1$ subsets $A_{v}$, then add $u$ to $\delta_{r}-k$ subsets not already containing $u$ (it does not matter which). As a result, each $u$ is contained in exactly $\delta_{r}$ subsets $A_{v}$.

Now define for each $v \in V$ the set $\mathcal{F}_{v}:=\left\{F \cap A_{v}: F \in \mathcal{F}\right\}$. Because $N_{G}^{r}[v] \subseteq A_{v}$, we have

$$
\left|\mathcal{F}_{v}\right| \leqslant\left|\mathcal{F}_{v, r}\right| \cdot 2^{\left|A_{v}\right|-\left|N_{G}^{r}[v]\right|} \leqslant 2^{\left|N_{G}^{r}[v]\right|} \rho \cdot 2^{\left|A_{v}\right|-\left|N_{G}^{r}[v]\right|} \leqslant 2^{\left|A_{v}\right|} \rho .
$$

Taking the product over all $v \in V$ and observing that $\sum_{v \in V}\left|A_{v}\right|=\delta_{r} n$, the claim follows by Lemma 4 .

To illustrate the use of Lemma 6 in a simple setting, let us reprove the Björklund et al. [7] upper bound for the number of connected sets of $G$ :

Corollary 7. Let $G$ be an n-vertex graph with maximum vertex degree at most $d$. Then $G$ has at most $\left(2^{d+1}-1\right)^{n /(d+1)}+n$ connected sets.

Proof. Let $\mathcal{F}$ be the family of connected sets of $G$, with the $n$ singleton sets consisting of each individual vertex removed from $\mathcal{F}$. Take $r=1$ and observe that then $\delta_{r}=d+1$. Furthermore, since the singleton sets $\{v\}$ have been removed from $\mathcal{F}$, we must have $F \cap N_{G}^{r}[v] \neq\{v\}$ for each $v \in V$ and $F \in \mathcal{F}$. It follows that we can take $\rho=1-1 / 2^{d+1}$ and the claim follows.

\subsection{Neighborhoods with Radius $r \geqslant 2$}

Let us now proceed to consider the case $r \geqslant 2$ and in particular the feasible projections of connected sets to a vertex neighborhood $N_{G}^{r}[v]$. Accordingly, assume that $r \geqslant 2$ is fixed.

Since our focus is on exponential growth rates as a function of the number of vertices, $n$, we can simplify the analysis by omitting all nonempty connected sets that are completely contained in at least one of the neighborhoods $N_{G}^{r}[v]$. Let us call such sets local connected sets. The following lemma is immediate.

Lemma 8. There are at most $\left(2^{\delta_{r}}-1\right) n$ local connected sets.

Our interest in what follows is thus to carry out a worst-case analysis of the number of connected sets that are not local. Let $\mathcal{F}$ be the family of non-local connected sets of $G$. Our intent is now to apply the projection method and Lemma 6 to $\mathcal{F}$. 


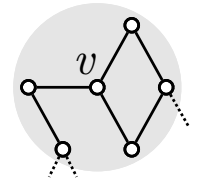

(a)

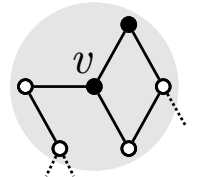

(d)

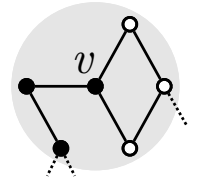

(b)

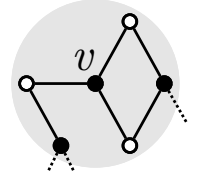

(e)

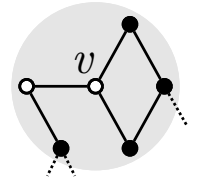

(c)

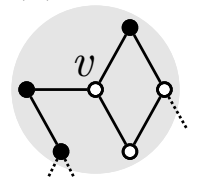

(f)

Figure 1: Vertex subsets that can $(\mathrm{a}-\mathrm{c})$ or cannot $(\mathrm{d}-\mathrm{f})$ belong to projections of non-local connected sets, with $d=3$ and $r=2$.

Intuitively, a connected set that is not local must "exit" any neighborhood that it intersects because otherwise the set would be localized in that neighborhood. In particular, such "exit" requires us to have vertices at the "boundary" of the neighborhood.

Let us say that a subset $S \subseteq N_{G}^{r}[v]$ is boundary-connected relative to $v$ if each connected component of $G[S]$ contains at least one vertex $u \in N_{G}^{r}[v] \backslash N_{G}^{r-1}[v]$ such that $u$ is adjacent to fewer than $d$ vertices in $N_{G}^{r}[v]$. (In particular, degree less than $d$ is necessary so that we can potentially "exit" from $u$ to outside $N_{G}^{r}[v]$. Note, however, that the definition does not require that such an exit actually exists in $G$. In particular we want this to be the case since we want to be able to check for boundary-connectivity without looking beyond the subgraph induced by $N_{G}^{r}[v]$.) Figure 1 shows examples of sets that are and are not boundary-connected for $d=3$ and $r=2$.

Lemma 9. Let $C$ be a non-local connected set of $G$. Then it holds for each vertex $v \in V$ that the projection $C \cap N_{G}^{r}[v]$ is boundary-connected relative to $v$.

Proof. When $C$ is empty the claim is trivial, so suppose that $C$ is nonempty. Because $C$ is non-local, we must have $C \nsubseteq N_{G}^{r}[v]$. It suffices to show that $S:=C \cap N_{G}^{r}[v]$ is boundaryconnected relative to $v$. Let $t \in C \backslash N_{G}^{r}[v]$. Let $G\left[S^{\prime}\right]$ be a connected component of $G[S]$ and $s \in S^{\prime}$. Because $C$ is a connected set, there is a path $\left(v_{0}, v_{1}, \ldots, v_{k}\right)$ in $G$ such that $v_{0}=s, v_{k}=t$ and, for some $i \leqslant k, s^{\prime}:=v_{i-1} \in S^{\prime}$ and $t^{\prime}:=v_{i} \in C \backslash S^{\prime}$. Now, $t^{\prime}$ cannot belong to $N_{G}^{r}[v]$, since otherwise we would get a contradiction to the assumption that $G\left[S^{\prime}\right]$ is a connected component of $G[S]$. Thus it holds that $s^{\prime} \in N_{G}^{r}[v] \backslash N_{G}^{r-1}[v]$. It remains to observe that $s^{\prime}$ is adjacent to at most $d-1$ vertices in $N_{G}^{r}[v]$, since $s^{\prime}$ is adjacent to $t^{\prime}$ and $G$ is of degree at most $d$.

Now observe that Lemma 9 and Lemma 6 together imply that the number of non-local connected sets of $G$ is bounded from above by $\left(2 \rho_{d, r}^{1 / \delta_{r}}\right)^{n}$, where $\rho_{d, r}$ is a constant such that every neighborhood $N_{G}^{r}[v]$ has at most $2^{\left|N_{G}^{r}[v]\right|} \rho_{d, r}$ boundary-connected sets $S$.

Our strategy for completing the proof of Theorem 1 is now to optimize the values $\rho_{d, r}$ for $r=2$ and $d \leqslant 5$ with computer search. Because boundary-connectivity is intrinsic 
to each neighborhood $N_{G}^{r}[v]$, we can carry out the optimization without paying attention how this neighborhood is connected to the rest of the graph.

\subsection{Extremal Neighborhood Graphs for $r=2$}

Let us say that a graph $H$ with maximum degree $d$ is a neighborhood graph with radius $r$ and root $v$ if the vertex set of $H$ is $N_{H}^{r}[v]$. Clearly, a neighborhood graph has at most $\delta_{r}$ vertices. Thus, for any fixed $d$ and $r$ we can optimize the constant $\rho_{d, r}$ by finding the maximum number of boundary-connected sets (relative to $v$ ) admitted by any neighborhood graph (with root $v$ ) for the parameters $d$ and $r$. This is what we proceed to do, using computer search.

The following small observation is useful to reduce the number of neighborhood graphs that need to be considered in the search.

Lemma 10. Let $H$ be a neighborhood graph with radius $r$ and root $v$. Let $H^{\prime}$ be the neighborhood graph with radius $r$ and root $v$ obtained from $H$ by removing each edge of $H$ that joins two vertices in $N_{H}^{r}[v] \backslash N_{H}^{r-1}[v]$. Then $H^{\prime}$ has at least as many boundaryconnected sets relative to $v$ as $H$.

Proof. Because all deleted edges join two vertices at maximum distance $(r)$ from $v$ in $H$, it is immediate that the $H^{\prime}$ is a neighborhood graph with radius $r$ and root $v$. Let $S$ be a boundary-connected set in $H$ relative to $v$. Because the removal of an edge leaves two vertices of degree less than $d$, any new connected component contains a vertex $u \in N_{H^{\prime}}^{r}[v] \backslash N_{H^{\prime}}^{r-1}[v]$ that is adjacent to fewer than $d$ vertices of $H^{\prime}$. Thus, $S$ is boundaryconnected in $H^{\prime}$ relative to $v$.

This lemma allows us to restrict our attention to neighborhood graphs in which the boundary vertices, that is, the set $N_{G}^{r}[v] \backslash N_{G}^{r-1}[v]$, form an independent set. We call these graphs essential neighborhood graphs.

Our focus on small parameters $r=2$ and $d \leqslant 5$ implies that the reduction to essential neighborhood graphs, combined with lightweight isomorph rejection suffices to carry out an optimization of $\rho_{d, r}$ with exhaustive search.

Let us now turn to the details of the algorithm that we use to enumerate the essential neighborhood graphs. Recall that we have fixed $r=2$ and the maximum degree to be at most $d \leqslant 5$. Suppose the graph $H$ has $n$ vertices. Since $r=2$ we can partition the set of vertices $V$ of $H$ into three sets $V_{0}, V_{1}, V_{2}$ based on distance from the root vertex $v \in V$. Let us write $\left|V_{0}\right|=n_{0},\left|V_{1}\right|=n_{1}$, and $\left|V_{2}\right|=n_{2}$. It is immediate that $V_{0}=\{v\}$ and hence $n_{0}=1$. Furthermore, $n=n_{0}+n_{1}+n_{2}$. Since the maximum degree is at most $d$, we have $1 \leqslant n_{1} \leqslant d$ and $1 \leqslant n_{2} \leqslant(d-1) n_{1}$. Thus in particular we observe that $3 \leqslant n \leqslant 1+d^{2}$. Finally, we observe that we can characterize the edges of $H$ as follows. First, each vertex in $V_{1}$ is adjacent to $v$. Second, the vertices in $V_{1}$ may or may not be adjacent to each other, we have to search through all possibilities within the degree bound. Third, each vertex in $V_{2}$ must be adjacent to at least one vertex in $V_{1}$ and must not be adjacent to $v$; again we have to search through all possibilities. Finally, because $H$ is an essential neighborhood graph, there are no edges joining the vertices in $V_{2}$. 


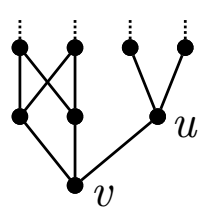

(a)

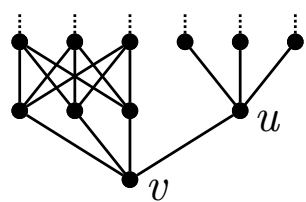

(b)

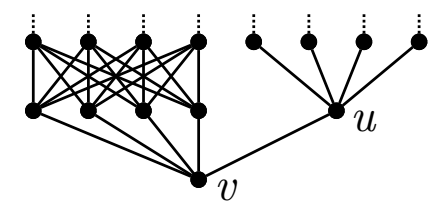

(c)

Figure 2: Worst-case neighborhood graphs of radius 2, for degree 3,4 , and 5 .

To reduce the number of isomorphic (and hence redundant) graphs encountered in the search, we implement the following lightweight isomorph rejection. Suppose that there is a total order on $V_{1}$ and on $V_{2}$. In the second stage of the algorithm, when we are searching through all possible ways of joining vertices in $V_{1}$ with edges, we require that the degrees of the vertices in $V_{1}$ form a non-increasing sequence if listed in the total order of $V_{1}$. Furthermore, in the third stage of the algorithm, when we are joining vertices in $V_{2}$ by edges to vertices in $V_{1}$, we require that with respect to the lexicographic order of subsets of $V_{1}$ it holds that $N_{H}[u] \leqslant N_{H}\left[u^{\prime}\right]$ whenever $u<u^{\prime}$ holds for $u, u^{\prime} \in V_{2}$. It is immediate that even with this isomorph rejection in place, the algorithm traverses at least one representative from every isomorphism class of neighborhood graphs (with the root individualized).

For each essential neighborhood graph $H$ that survives our isomorph rejection, we test whether the graph is not maximal, that is, whether it would be possible to add an edge with both ends in $V_{1}$ or an edge joining a vertex in $V_{1}$ with a vertex $V_{2}$ so that the affected vertices have degree at most $d$ in $V_{1}$ and at most $d-1$ in $V_{2}$ after the addition. If $H$ is not maximal, we reject it from further consideration. (Indeed, for a fixed value of $d$, a maximal graph maximizes the number of boundary-connected sets, and at least one such maximal graph from each isomorphism class of maximal graphs will be encountered in the search.)

Each $H$ that survives the maximality test is passed to a final enumeration of the boundary-connected sets, which proceeds as follows. First we add a new vertex $z$ to $H$ and join it by an edge to every vertex $u \in V_{2}$, provided that $u$ has degree less than $d$. Then we count the connected sets that contain $z$ using the folklore algorithm; see, for example, the description given by Björklund et al. [5].

The total time required to carry out the search was a few hours on a standard desktop computer with an Intel Core i7-4770K CPU. Figure 2 shows the worst-case neighborhood graphs found for $d=3,4,5$. The corresponding numbers of boundary-connected sets are 184, 1744, and 15136, respectively, yielding the optimal ratios

$$
\rho_{3,2}=184 / 2^{8}, \quad \rho_{4,2}=1744 / 2^{11}, \quad \rho_{5,2}=15136 / 2^{14} .
$$

To complete the proof of Theorem 1 it remains to apply Lemma 6 and calculate

$$
2 \rho_{3,2}^{1 / 10}=1.9350 \ldots, \quad 2 \rho_{4,2}^{1 / 17}=1.9811 \ldots, \quad 2 \rho_{5,2}^{1 / 26}=1.9939 \ldots
$$




\subsection{Limitations of the Method}

A fundamental limitation of neighborhood graphs and boundary-connectivity is that we have little control over what happens at the boundary vertices since these vertices may be connected beyond the boundary. With increasing $d$ or $r$ this limitation becomes more severe because the size of the boundary increases implying that we can exclude comparatively fewer and fewer projections when applying the projection method.

In fact, we can witness this limitation already for $r=2$ and $d \geqslant 6$ as we now proceed to demonstrate. Indeed, we observe that the worst-case neighborhood graphs shown in Figure 2 follow a pattern which we can generalize as follows:

Definition 11. An undirected graph $G$ is a d-mitten if its vertices can be partitioned into singletons $\{v\}$ and $\{u\}$ and sets $A, B, C$, each of size $d-1$ with exactly the following adjacencies: $v$ is adjacent to $u$ and every vertex in $A$. Every vertex in $A$ is adjacent to every vertex in $B$. Every vertex in $C$ is adjacent to $u$. The vertex $v$ is called the center of the $d$-mitten. The vertices $v$ and $u$ are shown in Figure 2.

We aim to show that any $d$-mitten has a large number of boundary-connected sets, and that particularly with $d \geqslant 6$ the number is large enough to only yield weak bounds for the number of connected sets. For any fixed $d$, this could be verified by direct calculation, possibly again aided by a computer. However, the simple structure of $d$-mittens allows us to find a closed-form expression that, not only enables the analysis for an arbitrary $d$, but also gives a way to check the correctness of the numbers computed for $d=3,4,5$ by the general algorithm.

Lemma 12. Let $G$ be a d-mitten with center $v$. Then the number of boundary-connected sets of $G$ relative to $v$ is given by $2^{3 d-1}-5 \cdot 2^{2 d-2}+2^{d}$.

Proof. Let $\{u\}, A, B$, and $C$ be the vertex subsets of $G$ guaranteed in the definition of $d$-mitten. We will count the number of vertex subsets $S$ of $G$ that are not boundaryconnected relative to $v$, or n.b.c. for short. We consider four disjoint cases and denote their contributions to the count by $c_{1}, c_{2}, c_{3}$, and $c_{4}$.

Assume first that $v \in S$. Observe that now $S$ is n.b.c. if and only if there is no path in $G[S]$ from $v$ to a vertex in $B$ or $C$. We consider separately the cases $u \in S$ and $u \notin S$. Suppose $u \in S$. Then $S$ cannot intersect $C$, since otherwise there would be a path in $G[S]$ from $v$ to a vertex in $C$. Likewise, $S$ can intersect only $A$ or $B$ but not both. Now, if $S$ does not intersect $A$, then $S$ may contain any of the $2^{d-1}$ subsets of $B$, and vice versa, yielding $c_{1}:=2^{d}-1$ possibilities for $S$ in total, where the -1 is due to double counting the case where $S$ is disjoint from both $A$ and $B$. Suppose then that $u \notin S$. Again, we have $2^{d}-1$ possible intersections with $A \cup B$, but now, in addition, any subset of $C$ can be contained in $S$, yielding $c_{2}:=\left(2^{d}-1\right) 2^{d-1}$ possibilities for $S$ in total.

Assume then that $v \notin S$. Now $S$ is n.b.c. if and only if $X:=S \cap(\{u\} \cup C)$ is n.b.c. or $Y:=S \cap(A \cup B)$ is n.b.c. We count first the cases where $X$ is n.b.c. This holds exactly when $u \in S$ and $S$ does not intersect $C$. From $A$ and $B$ any subset can be contained in $S$, yielding $c_{3}:=2^{2(d-1)}$ possibilities. Finally, we count the cases where $Y$ is n.b.c. but 


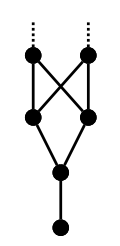

(a)

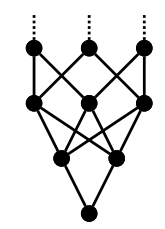

(b)

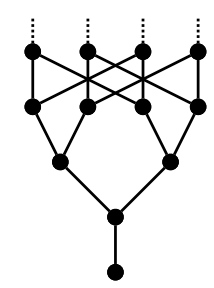

(c)

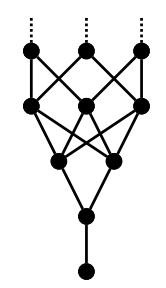

(d)

Figure 3: Neighborhood graphs with a large number of boundary-connected sets.

$X$ is not. The set $Y$ is n.b.c. exactly when $S$ intersects $A$ but not $B$, yielding $2^{d-1}-1$ possibilities. The set $X$ has $2^{d}$ possible configurations of which exactly one is n.b.c. Thus, we have $c_{4}:=\left(2^{d-1}-1\right)\left(2^{d}-1\right)$ possibilities in total.

Summing up $c_{1}+c_{2}+c_{3}+c_{4}$ yields $5 \cdot 2^{2 d-2}-2^{d}$. It remains to note that $G$ has $3 d-1$ vertices and thus $2^{3 d-1}$ vertex subsets in total.

The following lemma shows that $d$-mittens, indeed, result in weak bounds compared to the simple bound:

Lemma 13. Let $d \geqslant 6$. Then

$$
\left(2^{d+1}-1\right)^{1 /(d+1)} \leqslant 2\left(\frac{2^{3 d-1}-5 \cdot 2^{2 d-2}+2^{d}}{2^{3 d-1}}\right)^{1 /\left(d^{2}+1\right)} .
$$

Proof. For $d=6$, the inequality is verified by direct calculation (details omitted).

Suppose $d \geqslant 7$. Multiplying both sides by $2^{-1}$ and raising to the $d^{2}+1$, the left-hand side becomes $2^{-\left(d^{2}+1\right)} \cdot\left(2^{d+1}-1\right)^{\left(d^{2}+1\right) /(d+1)}$. Simplifying this product and the remaining fraction on the right-hand side, we have that the inequality holds if and only if

$$
\left(1-2^{-d-1}\right)^{\left(d^{2}+1\right) /(d+1)} \leqslant 1-5 \cdot 2^{-d-1}+2^{-2 d+1} .
$$

We proceed by applying the Bonferroni inequality

$$
(1-p)^{k} \leqslant 1-k p+\left(\begin{array}{l}
k \\
2
\end{array}\right) p^{2}
$$

with $p:=2^{-d-1}$ and $k:=\left\lfloor\left(d^{2}+1\right) /(d+1)\right\rfloor$. Observe that $\left(\begin{array}{c}k \\ 2\end{array}\right) \leqslant\left(\begin{array}{c}d+1 \\ 2\end{array}\right) \leqslant 2^{d+1}$ and $k \geqslant\left\lfloor\left(7^{2}+1\right) /(7+1)\right\rfloor=6$. Thus, we have $(1-p)^{k} \leqslant 1-(k-1) p \leqslant 1-5 \cdot 2^{-d-1}$, completing the proof.

Finally, we turn to the case where the radius $r$ is larger than 2. Here we only investigate the cases where $r$ equals 3 or 4 and the maximum degree $d$ equals 3 and 4 . For these cases, the graphs shown in Figure 3 imply

$$
\rho_{3,3} \geqslant 31 / 2^{6}, \quad \rho_{4,3} \geqslant 321 / 2^{9}, \quad \rho_{3,4} \geqslant 1480 / 2^{12}, \quad \rho_{4,4} \geqslant 459 / 2^{10} .
$$

Consequently,

$$
2 \rho_{3,3}^{1 / 22} \geqslant 1.9351, \quad 2 \rho_{4,3}^{1 / 53} \geqslant 1.9824, \quad 2 \rho_{3,4}^{1 / 46} \geqslant 1.9562, \quad 2 \rho_{4,4}^{1 / 161} \geqslant 1.9900,
$$

exceeding the respective values $b_{3}=1.9351$ and $b_{4}=1.9812$ given in Theorem 1 . 

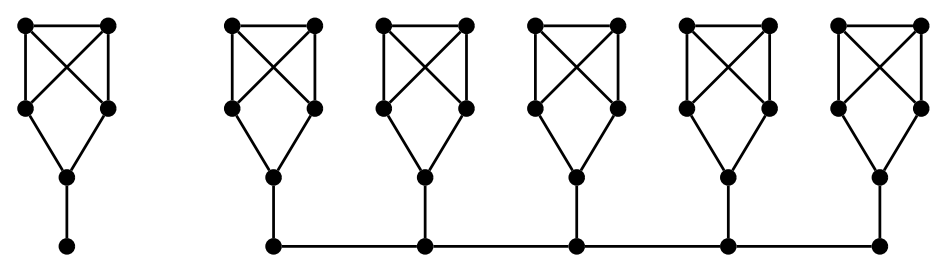

Figure 4: A gadget $G$ of degree 3 (left) and the graph $G_{5}$ (right) consisting of five gadgets linked together by their join vertices.

\section{Lower Bounds on the Number of Connected Sets}

In this section we seek lower bounds on the maximum number of connected sets in graphs of bounded degree. As noted by Perrier et al. [26] in an empirical analysis, graphs that maximize the number of connected sets for a fixed number of vertices tend to resemble trees locally; in other words, they are regular graphs of high girth and a diameter logarithmic in the number of vertices. While considering such graphs directly thus seems promising, they appear to be tricky to both construct and analyze. Indeed, we are not aware of any infinite family of regular graphs of unbounded girth or sublinear diameter where the number of connected sets would admit a simple closed-form expression.

On the other hand, the number of connected sets can be recursively characterized in several graph families where the graphs are obtained by chaining together "gadgets" of bounded size. While the diameter of such graphs scales linearly in the number of gadgets (and thus in the number of vertices), it turns out that using sufficiently large gadgets of high girth is already enough to produce good lower bounds.

We prove Theorem 2 by considering two types of gadget constructions in Sections 3.1 and 3.2. In Section 3.3 we extend the construction to degrees larger than 5, proving Theorem 3.

\subsection{Gadgets of Type I and II}

We start by considering a very simple construction where gadgets are joined to each other along a path. Let $G$ be a $k$-vertex graph of degree $d$, containing one special vertex of degree at most $d-2$, called the join vertex. We call $G$ a $(d, k)$-gadget of type $I$. We can use $G$ to construct an infinite family of graphs of degree $d$ as follows: For each $t=1,2, \ldots$, let $G_{t}$ be the graph obtained by taking the union of $t$ copies of $G$ and adding $t-1$ edges between the join vertices so that they induce a path (see Figure 4). To analyze the asymptotic growth of the number of connected sets in the family $G_{1}, G_{2}, \ldots$ it suffices to consider connected sets that include all join vertices. To that end, let $c$ be the number of connected sets of $G$ that include the join vertex of $G$. Then, $G_{t}$ has at least $c^{t}=a^{n}$ connected sets, where $a=c^{1 / k}$ and $n=t k$ is the number of vertices in $G_{t}$. Obtaining good lower bounds thus comes down to finding gadgets where $a$ is large.

As a second construction, we consider linking gadgets together by a single edge. Let $G$ be a $k$-vertex graph of degree $d$ with four labeled vertices $u, v, u^{\prime}$ and $v^{\prime}$. Let $G$ contain 

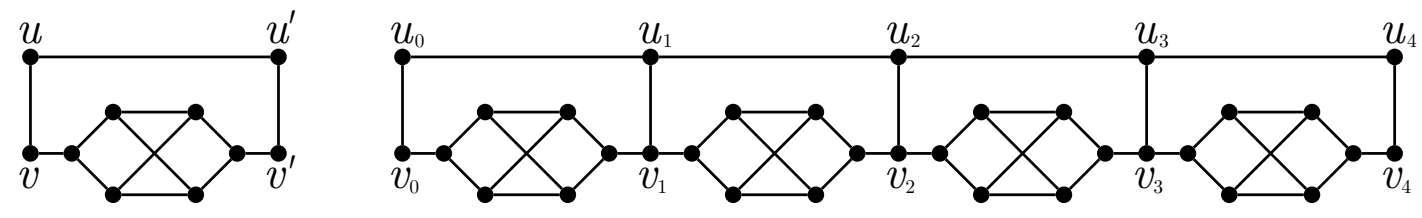

Figure 5: A $(3,10)$-gadget $G$ of Type II (left) and the graph $G_{4}$ (right) consisting of four gadgets linked together.

the edges $\{u, v\}$ and $\left\{u^{\prime}, v^{\prime}\right\}$ and any other edges such that

$$
\operatorname{deg}(u)+\operatorname{deg}\left(u^{\prime}\right) \leqslant d-1 \quad \text { and } \quad \operatorname{deg}(v)+\operatorname{deg}\left(v^{\prime}\right) \leqslant d-1 .
$$

We call $G$ a $(d, k)$-gadget of type $I I$. We now construct an infinite family of graphs by chaining gadgets so that for each gadget the vertices $u^{\prime}$ and $v^{\prime}$ are merged with the vertices $u$ and $v$ of the next gadget, respectively (see Figure 5 ). Formally, for all $t=1,2, \ldots$ we define the graph $G_{t}$ recursively as follows: Let $G_{0}$ be the graph consisting of exactly two adjacent vertices, labeled $u_{0}$ and $v_{0}$. For $t>0$ start by setting $G_{t}$ to be the union of $G_{t-1}$ and $G$. Then modify $G_{t}$ by making $u_{t-1}$ adjacent to every neighbor of $u$, and $v_{t-1}$ adjacent to every neighbor of $v$. Note that it follows from condition (1) that adding the edges will not result in vertex degrees greater than $d$. Afterwards remove both $u$ and $v$ from $G_{t}$, and finally relabel the vertices $u^{\prime}$ and $v^{\prime}$ to be $u_{t}$ and $v_{t}$, respectively, so that the labeled vertices in $G_{t}$ will be $u_{0}, u_{1}, \ldots, u_{t}$ and $v_{0}, v_{1}, \ldots, v_{t}$.

We now characterize the number of connected sets of $G_{t}$ recursively. For all $t=0,1, \ldots$ let $\mathcal{C}_{t}$ denote the family of connected sets of $G_{t}$ that intersect $\left\{u_{i}, v_{i}\right\}$ for all $i=0,1, \ldots, t$. Partition $\mathcal{C}_{t}$ into three sets $\mathcal{X}_{t}, y_{t}$, and $z_{t}$ as follows. Let $X_{t}$ contain the sets containing both $u_{t}$ and $v_{t}, y_{t}$ the sets containing $u_{t}$ but not $v_{t}$, and $z_{t}$ the sets containing $v_{t}$ but not $u_{t}$. Denoting the sizes of these set families by $x_{t}, y_{t}, z_{t}$, we have that $x_{0}=y_{0}=z_{0}=1$, and for all $t \geqslant 1$ the sizes can be obtained as a linear combination of the sizes for $t-1$. Specifically, denote by $c_{A}$ the number of connected sets $C$ of $G$ such that $\left\{u, v, u^{\prime}, v^{\prime}\right\} \cap C=A$, and define the matrix

$$
M_{G}:=\left[\begin{array}{ccc}
c_{\left\{u, v, u^{\prime}, v^{\prime}\right\}} & c_{\left\{u, u^{\prime}, v^{\prime}\right\}} & c_{\left\{v, u^{\prime}, v^{\prime}\right\}} \\
c_{\left\{u, v, u^{\prime}\right\}} & c_{\left\{u, u^{\prime}\right\}} & c_{\left\{v, u^{\prime}\right\}} \\
c_{\left\{u, v, v^{\prime}\right\}} & c_{\left\{u, v^{\prime}\right\}} & c_{\left\{v, v^{\prime}\right\}}
\end{array}\right] .
$$

Observe that the columns of $M_{G}$ correspond to combinations of $u$ and $v$ (except the empty combination) and the rows correspond to combinations of $u^{\prime}$ and $v^{\prime}$. Then, for $t \geqslant 1$ we have that

$$
\left[\begin{array}{l}
x_{t} \\
y_{t} \\
z_{t}
\end{array}\right]=M_{G}\left[\begin{array}{l}
x_{t-1} \\
y_{t-1} \\
z_{t-1}
\end{array}\right] \text {, and thus }\left[\begin{array}{l}
x_{t} \\
y_{t} \\
z_{t}
\end{array}\right]=M_{G}^{t}\left[\begin{array}{l}
1 \\
1 \\
1
\end{array}\right] \text {. }
$$

To analyze this recurrence, we make the sensible assumption that $G$ is connected. For now, assume also that there is a path from both $u$ and $v$ to $\left\{u^{\prime}, v^{\prime}\right\}$ that does not use the edge $\{u, v\}$. Symmetrically, assume that there is a path from both $u^{\prime}$ and $v^{\prime}$ to $\{u, v\}$ 

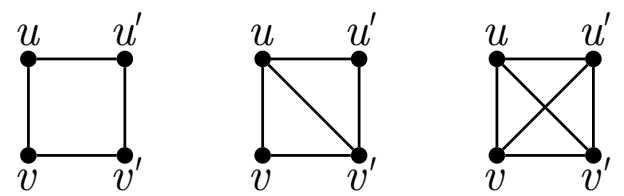

Figure 6: The smallest gadgets of type II, $L_{3}$ (left), $L_{4}$ (middle), and $L_{5}$ (right), which can be concatenated to produce the generalized ladder graphs.

not using the edge $\left\{u^{\prime}, v^{\prime}\right\}$. We say that $G$ is nice if it has these properties and prove the following lemma.

Lemma 14. If $G$ is nice, then $M_{G}$ is primitive, that is, there is an integer $t>0$ such that $M_{G}^{t}$ is (strictly) positive.

Proof. Since $\left\{u^{\prime}, v^{\prime}\right\}$ is reachable from both $u$ and $v$ without using the edge $\{u, v\}$, we see that all entries of the first row of $M_{G}$ are positive. By the symmetrical assumption the entries on the first column are likewise positive. It is then easy to see that all entries of $M_{G}^{2}$ are positive.

For a primitive matrix $A$, the Perron-Frobenius theorem guarantees that $A$ has an eigenvalue $\lambda_{A}$ that is real and determines the asymptotic behavior of $A^{t}$, that is, we have $\lim _{t \rightarrow \infty} A^{t} / \lambda_{A}^{t}=P$ for a positive matrix $P$. It follows that $\left|\mathcal{C}_{t}\right|=x_{t}+y_{t}+z_{t}=\Theta\left(\lambda_{M_{G}}^{t}\right)=$ $\Theta\left(a^{n}\right)$, where $a=\lambda_{M_{G}}^{1 /(k-2)}$ and $n$ is the number of vertices in $G_{t}$. Computing $M_{G}$ and the number $a$ for a given gadget $G$ thus comes down to enumerating all connected sets of $G$.

To conclude the analysis, consider the case when $G$ is not nice. Then it has at least one row of zeroes and thus $M_{G}^{t}$ also has a column of zeroes for all $t \geqslant 2$. We may thus discard the zero row and column, and the asymptotic behavior is then given by the dominant eigenvalue of the remaining $2 \times 2$ matrix.

For each gadget $G$ of type I or II, we denote by $g(G)$ the base of the asymptotic lower bound that is implied by $G$, that is, we will have $\left|\mathcal{C}_{t}\right|=\Omega\left(g(G)^{n}\right)$. To prove Theorem 2 it remains to construct appropriate gadgets for each degree bound $d$.

\subsection{Lower Bounds for Individual Degrees}

To illustrate the use of the gadgets, let us first reprove the previous lower bounds for $d \leqslant 5$ based on generalized ladder graphs. The ladder graphs are a special case of constructions of type II; they are produced by the three smallest gadgets, $L_{3}, L_{4}$, and $L_{5}$, illustrated in Figure 6. The corresponding matrices are

$$
M_{L_{3}}=\left[\begin{array}{lll}
1 & 1 & 1 \\
1 & 1 & 0 \\
1 & 0 & 1
\end{array}\right], \quad M_{L_{4}}=\left[\begin{array}{lll}
1 & 1 & 1 \\
1 & 1 & 0 \\
1 & 1 & 1
\end{array}\right], \quad M_{L_{5}}=\left[\begin{array}{lll}
1 & 1 & 1 \\
1 & 1 & 1 \\
1 & 1 & 1
\end{array}\right] \text {, }
$$

and computing their eigenvalues yields the lower bounds $g\left(L_{3}\right) \geqslant 1.5537, g\left(L_{4}\right) \geqslant 1.6180$ and $g\left(L_{5}\right) \geqslant 1.7320$. 


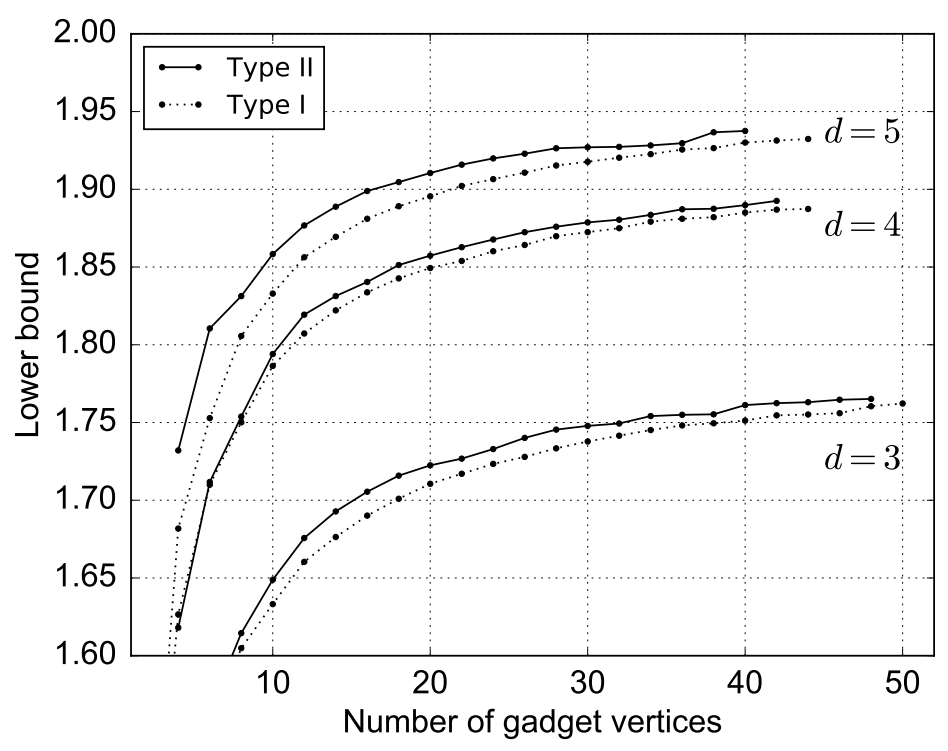

Figure 7: The development of the best lower bounds found as the gadget size $k$ grows. The results are shown here only for even $k$ as gadgets with an odd number of vertices are always non-regular for some $d$ and typically result in relatively worse bounds.

It turns out that larger gadgets of high girth yield significantly better lower bounds than the three simple gadgets. To investigate the relationship between gadgets and the quality of the resulting bounds in depth, we carry out a computer search over gadgets of type I and II. Since the number of possible gadgets grows very rapidly, we are able to enumerate them only up to a very small number of vertices. For larger gadget sizes we instead sample multiple gadgets and aim to optimize them via local search techniques such as greedy hillclimbing with random restarts. We terminate the search at a size where evaluating the asymptotic lower bound for a single gadget would require several days.

Figure 7 shows the improvement in the best lower bounds found for degree $d \in\{3,4,5\}$ as the gadget size grows. We observe that the bounds grow at a similar pace for both gadget types and that gadgets of type II generally result in slightly better bounds. For the explored range of gadgets (up to 50 vertices) there is no indication that the bounds would reach a global optimum at a certain gadget size; rather, the results suggest that the bounds might become increasingly better as gadgets become larger and more complex. Similar observations can be made for gadgets of degree $d>5$.

For all $d \geqslant 3$, we denote by $B_{d}$ the best gadget of degree $d$ found in the search. These gadgets are all of type II. The respective lower bounds $g\left(B_{d}\right)$ are shown in Table 1 for small values of $d$, in comparison to the previous best lower bounds and the best known upper bounds. This completes the proof of Theorem 2.

The gadgets $B_{d}$ for $d \in\{3,4,5\}$ and the corresponding matrices $M_{B_{d}}$ are presented in the appendix. In the online appendix we further present all gadgets shown in Figure 7 , gadgets for higher vertex degrees, as well as any better gadgets discovered in the future. 
Table 1: Bases of exponential asymptotic lower and upper bounds on the number of connected sets in graphs of degree at most $d$.

\begin{tabular}{ccccccc}
\hline \multirow{2}{*}{ Degree bound } & \multicolumn{3}{c}{ Lower bound } & Estimate & \multicolumn{2}{c}{ Upper bound } \\
$d$ & $g\left(C_{d}\right)$ & $g\left(L_{d}\right)$ & $g\left(B_{d}\right)$ & $\gamma_{d}[26]$ & $b_{d}$ & $\beta_{d}[7]$ \\
\hline 3 & 1.4142 & 1.5537 & 1.7651 & 1.81 & 1.9351 & 1.9680 \\
4 & 1.5874 & 1.6180 & 1.8925 & 1.92 & 1.9812 & 1.9874 \\
5 & 1.6817 & 1.7320 & 1.9375 & 1.96 & 1.9940 & 1.9948 \\
6 & 1.7411 & 1.8171 & 1.9577 & & & 1.9978 \\
7 & 1.7817 & 1.8612 & 1.9662 & & & 1.9991 \\
8 & 1.8114 & 1.8881 & 1.9734 & & & 1.9996 \\
\hline
\end{tabular}

\subsection{Lower Bounds for the General Degree}

While searching for good gadgets can yield improved lower bounds for any fixed vertex degree, devising general constructions that yield a bound for all $d \geqslant 3$ appears to be more challenging. As we are not aware of any such general bounds in the literature, we present two simple constructions based on gadgets of type I and type II.

For gadgets of type I, consider the following construction for an arbitrary degree $d \geqslant 3$. Let $C_{d}$ be the complete graph on $d-1$ vertices, with an arbitrary vertex (of degree $d-2$ ) chosen as the join vertex. Then $C_{d}$ has $2^{d-2}$ connected sets that contain the join vertex, and we thus have the lower bound $g\left(C_{d}\right)=2^{(d-2) /(d-1)}$.

With gadgets of type II, we obtain a bound for all $d \geqslant 3$ by generalizing the ladder graphs further as follows. For $d>5$, define $L_{d}$ by taking $L_{5}$ and adding a complete graph on new vertices $u_{1}, u_{2}, \ldots, u_{d-5}$ as well as the edges $\left\{u, u_{i}\right\}$ and $\left\{v, u_{i}\right\}$ for all $1 \leqslant i \leqslant d-5$. Counting the connected sets of $L_{d}$ that contain $u, v$, or both, we may take any combination of the added $d-5$ vertices and the bound $g\left(L_{d}\right)=\left(3 \cdot 2^{d-5}\right)^{1 /(d-3)}$ easily follows without constructing the matrix $M_{L_{d}}$. This completes the proof of Theorem 3.

Values of $g\left(C_{d}\right)$ and $g\left(L_{d}\right)$ are shown in Table 1 for small values of $d$. We note that searching for good gadgets for individual $d \geqslant 6$ will easily yield better lower bounds than either of the general bounds.

\section{Concluding Remarks}

This paper has explored the possibility of extending the projection method to neighborhoods with radius $r \geqslant 2$ to obtain improved upper bounds for the number of connected sets in bounded degree graphs. Our improved bounds for $d \leqslant 5$ present a rather modest improvement, and there remains a significant gap between the upper and lower bounds in Theorems 1 and 2. To strengthen the projection method it would appear that one needs control on how the projections change as one moves from one neighborhood to the 
neighborhood of an adjacent vertex. To improve upon the lower bounds, one possible avenue is to expand the search towards larger gadgets. To cope with the computational challenge of evaluating the gadgets, it might be possible to resort to approximations or to restrict focus to a more regular subset of gadgets, where computing the number of connected sets would be easier.

\section{Acknowledgements}

We wish to thank the reviewers for bringing a wealth of relevant literature to our attention as well as for helpful suggestions for improving the presentation.

\section{References}

[1] A. Agrawal, S. Gupta, S. Saurabh, and R. Sharma. Improved algorithms and combinatorial bounds for independent feedback vertex set. In 11th International Symposium on Parameterized and Exact Computation, IPEC 2016, volume 63 of LIPIcs, pages 2:1-2:14. Schloss Dagstuhl - Leibniz-Zentrum für Informatik, 2016.

[2] N. Alon. Independent sets in regular graphs and sum-free subsets of finite groups. Israel Journal of Mathematics, 73:247-256, 1991.

[3] D. Binkele-Raible, L. Brankovic, M. Cygan, H. Fernau, J. Kneis, D. Kratsch, A. Langer, M. Liedloff, M. Pilipczuk, P. Rossmanith, and J. O. Wojtaszczyk. Breaking the $2^{\mathrm{n}}$-barrier for irredundance: Two lines of attack. Journal of Discrete Algorithms, 9(3):214-230, 2011.

[4] D. Binkele-Raible, H. Fernau, S. Gaspers, and M. Liedloff. Exact and parameterized algorithms for max internal spanning tree. Algorithmica, 65(1):95-128, 2013.

[5] A. Björklund, T. Husfeldt, P. Kaski, and M. Koivisto. Computing the Tutte polynomial in vertex-exponential time. In 49th Annual IEEE Symposium on Foundations of Computer Science, FOCS 2008, pages 677-686. IEEE Computer Society, 2008.

[6] A. Björklund, T. Husfeldt, P. Kaski, and M. Koivisto. Trimmed Moebius inversion and graphs of bounded degree. Theory of Computing Systems, 47(3):637-654, 2010.

[7] A. Björklund, T. Husfeldt, P. Kaski, and M. Koivisto. The traveling salesman problem in bounded degree graphs. ACM Transactions on Algorithms, 8(2):18:1-13, 2012.

[8] B. Bollobás. The Art of Mathematics: Coffee Time in Memphis. Cambridge University Press, 2006.

[9] J. M. Byskov, B. A. Madsen, and B. Skjernaa. On the number of maximal bipartite subgraphs of a graph. Journal of Graph Theory, 48(2):127-132, 2005.

[10] F. Chung, R. Graham, P. Frankl, and J. Shearer. Some intersection theorems for ordered sets and graphs. Journal of Combinatorial Theory, Series A, 43(1):23-37, 1986. 
[11] F. V. Fomin, S. Gaspers, A. V. Pyatkin, and I. Razgon. On the minimum feedback vertex set problem: Exact and enumeration algorithms. Algorithmica, 52(2):293-307, 2008.

[12] F. V. Fomin, F. Grandoni, A. V. Pyatkin, and A. A. Stepanov. Combinatorial bounds via measure and conquer: Bounding minimal dominating sets and applications. $A C M$ Transactions on Algorithms, 5(1):9:1-17, 2008.

[13] F. V. Fomin and Y. Villanger. Finding induced subgraphs via minimal triangulations. In 27th International Symposium on Theoretical Aspects of Computer Science, STACS 2010, volume 5 of LIPIcs, pages 383-394. Schloss Dagstuhl - Leibniz-Zentrum für Informatik, 2010.

[14] D. Galvin. An upper bound for the number of independent sets in regular graphs. Discrete Mathematics, 309(23-24):6635-6640, 2009.

[15] S. Gaspers, D. Kratsch, and M. Liedloff. On independent sets and bicliques in graphs. Algorithmica, 62(3-4):637-658, 2012.

[16] S. Gaspers and S. Mackenzie. On the number of minimal separators in graphs. Journal of Graph Theory, 87(4):653-659, 2018.

[17] S. Gaspers and M. Mnich. Feedback vertex sets in tournaments. Journal of Graph Theory, 72(1):72-89, 2013.

[18] P. A. Golovach, P. Heggernes, and D. Kratsch. Enumerating minimal connected dominating sets in graphs of bounded chordality. Theoretical Computer Science, 630:63-75, 2016.

[19] P. A. Golovach, P. Heggernes, and D. Kratsch. Enumeration and maximum number of minimal connected vertex covers in graphs. European Journal of Combinatorics, 68:132-147, 2018.

[20] S. Gupta, V. Raman, and S. Saurabh. Maximum r-regular induced subgraph problem: Fast exponential algorithms and combinatorial bounds. SIAM Journal on Discrete Mathematics, 26(4):1758-1780, 2012.

[21] J. Kahn. An entropy approach to the hard-core model on bipartite graphs. Combinatorics, Probability and Computing, 10:219-237, 2001.

[22] K. Kangas, P. Kaski, M. Koivisto, and J. H. Korhonen. On the number of connected sets in bounded degree graphs. In 40th International Workshop on Graph-Theoretic Concepts in Computer Science, WG 2014. Revised Selected Papers, volume 8747 of Lecture Notes in Computer Science, pages 336-347. Springer, 2014.

[23] R. E. Miller and D. E. Muller. A problem of maximum consistent subsets. IBM research report RC-240, J.T. Technical report, Watson Research Center, 1960.

[24] M. Mnich and E. Teutrine. Improved bounds for minimal feedback vertex sets in tournaments. In 11th International Symposium on Parameterized and Exact Computation, IPEC 2016, volume 63 of LIPICs, pages 24:1-24:10. Schloss Dagstuhl Leibniz-Zentrum für Informatik, 2016. 
[25] J. W. Moon and L. Moser. On cliques in graphs. Israel Journal of Mathematics, 3:23-28, 1965.

[26] E. Perrier, S. Imoto, and S. Miyano. Finding optimal Bayesian network given a super-structure. Journal of Machine Learning Research, 9:2251-2286, 2008.

[27] M. Pilipczuk and M. Pilipczuk. Finding a maximum induced degenerate subgraph faster than $2^{\mathrm{n}}$. In 7th International Symposium on Parameterized and Exact Computation, IPEC 2012, volume 7535 of Lecture Notes in Computer Science, pages 3-12. Springer, 2012.

[28] A. Vince. Counting connected sets and connected partitions of a graph. Australasian Journal of Combinatorics, 67:281-293, 2017.

[29] Y. Zhao. The number of independent sets in a regular graph. Combinatorics, Probability and Computing, 19:315-320, 2010.

\section{Appendix}

We specify here the type II gadgets $B_{d}$ that result in the current best lower bounds for degree $d \in\{3,4,5\}$. The vertices are indexed from 0 to $n-1$, and the indices $0,1,2,3$ represent the labeled vertices $u, v, u^{\prime}, v^{\prime}$, respectively. We list explicitly all adjacencies between the vertices and also display the matrix $M_{B_{d}}$.

\section{$B_{3}, 48$ vertices}

\begin{tabular}{|c|c|c|c|c|c|c|c|c|c|c|c|}
\hline $0-1$ & $4-16$ & $6-15$ & 8-31 & $10-45$ & $12-46$ & $15-18$ & 19-33 & $21-37$ & $25-27$ & 29-39 & $39-40$ \\
\hline $0-4$ & $4-37$ & $6-47$ & $8-35$ & $11-14$ & $13-27$ & $15-23$ & $19-47$ & $21-41$ & $25-36$ & $30-40$ & $39-42$ \\
\hline $1-19$ & $5-23$ & $7-17$ & $9-17$ & $11-16$ & $13-41$ & 16-38 & 20-31 & $22-25$ & $26-30$ & $33-36$ & $42-47$ \\
\hline $2-3$ & $5-27$ & $7-34$ & $9-22$ & $11-28$ & $13-43$ & $17-31$ & $20-38$ & $22-28$ & $26-44$ & $34-35$ & $43-45$ \\
\hline $2-42$ & $5-30$ & $7-45$ & $9-32$ & $12-23$ & $14-26$ & $18-35$ & $20-40$ & $24-32$ & $29-32$ & $37-46$ & \\
\hline $3-24$ & $6-10$ & $8-28$ & $10-36$ & $12-44$ & $14-43$ & $18-44$ & 21-33 & $24-46$ & 29-34 & $38-41$ & \\
\hline & & & & 746955 & $\begin{array}{l}38329 \\
38676 \\
17007\end{array}$ & $\begin{array}{l}373234 \\
393977\end{array}$ & $\begin{array}{l}236 \\
959 \\
7131\end{array}$ & 3660586 & $\begin{array}{l}080 \\
037 \\
013\end{array}$ & & \\
\hline
\end{tabular}

\section{$B_{4}, 42$ vertices}

$\begin{array}{llllllllllll}0-1 & 4-7 & 5-41 & 7-36 & 9-40 & 11-35 & 13-33 & 15-37 & 19-38 & 22-26 & 24-39 & 30-39 \\ 0-2 & 4-9 & 6-20 & 8-16 & 10-15 & 11-36 & 13-37 & 16-35 & 20-26 & 22-30 & 26-38 & 31-41 \\ 1-29 & 4-23 & 6-25 & 8-18 & 10-19 & 12-13 & 14-25 & 17-19 & 20-34 & 22-35 & 27-31 & 32-33 \\ 2-3 & 4-29 & 6-30 & 8-36 & 10-27 & 12-19 & 14-27 & 17-21 & 20-35 & 23-34 & 27-32 & 34-41 \\ 2-41 & 5-25 & 6-40 & 8-38 & 10-36 & 12-31 & 14-34 & 17-23 & 21-28 & 24-26 & 28-31 & \\ 3-16 & 5-32 & 7-21 & 9-15 & 11-28 & 12-39 & 14-38 & 18-23 & 21-30 & 24-33 & 28-40 & \\ 3-39 & 5-40 & 7-32 & 9-18 & 11-29 & 13-17 & 15-16 & 18-37 & 22-25 & 24-37 & 29-33 & \end{array}$




$$
M_{B_{4}}=\left[\begin{array}{rrr}
74052300417 & 49548111633 & 25941109433 \\
63360678728 & 30081845289 & 0 \\
33177340176 & 15750317593 & 25941109433
\end{array}\right]
$$

\section{$B_{5}, 40$ vertices}

$\begin{array}{llllllllllll}0-1 & 3-17 & 5-26 & 7-22 & 9-14 & 11-23 & 13-25 & 16-24 & 19-20 & 22-25 & 24-38 & 29-34 \\ 0-3 & 4-5 & 5-30 & 7-23 & 9-19 & 11-25 & 13-32 & 16-37 & 19-33 & 22-30 & 25-39 & 29-35 \\ 0-25 & 4-10 & 6-8 & 7-28 & 9-35 & 11-30 & 13-39 & 16-39 & 20-27 & 23-29 & 26-32 & 30-35 \\ 1-13 & 4-12 & 6-9 & 7-39 & 10-15 & 11-31 & 14-18 & 17-26 & 20-31 & 23-36 & 26-33 & 31-35 \\ 1-15 & 4-13 & 6-14 & 8-15 & 10-18 & 12-19 & 14-28 & 17-34 & 20-34 & 23-38 & 27-32 & 32-37 \\ 2-3 & 4-19 & 6-21 & 8-18 & 10-21 & 12-21 & 14-33 & 17-37 & 20-38 & 24-31 & 27-38 & 33-37 \\ 2-9 & 5-16 & 6-32 & 8-34 & 10-27 & 12-31 & 15-30 & 18-26 & 21-27 & 24-33 & 28-29 & 34-36 \\ 2-28 & 5-22 & 7-15 & 8-35 & 11-17 & 12-39 & 16-22 & 18-36 & 21-29 & 24-36 & 28-38 & 36-37 \\ & M_{B_{5}}=\left[\begin{array}{lllllll}40571248665 & 25343886998 & 35402994593 \\ 36131010078 & 13876386956 & 26714905772 \\ 26168254403 & 21595708566 & 13917032744\end{array}\right] & & & \end{array}$

